\title{
Avaliação da função pulmonar de recém-nascidos com síndrome do desconforto respiratório em diferentes pressões finais expiratórias positivas
}

\author{
Assessment of pulmonary function of preterm newborn infants \\ with respiratory distress syndrome at different positive end expiratory pressure levels
}

Luiz C.T. Consolo ${ }^{1}$, Durval B. Palhares ${ }^{2}$, Lourdes Z.Z. Consolo ${ }^{3}$

\section{Resumo}

Objetivos: verificar as alterações da função pulmonar: complacência dinâmica $(\mathrm{Cdyn})$, volume corrente inspiratório $\left(\mathrm{V}_{\text {Tinsp }}\right)$, pressão arterial de dióxido de carbono $\left(\mathrm{PaCO}_{2}\right)$, em recém-nascidos prétermo com síndrome do desconforto respiratório.

Pacientes e métodos: estudo de caso controle, incluindo 11 prétermos com idade gestacional $<35$ semanas, e com peso de nascimento $<2.500$ gramas, todos com diagnóstico de síndrome do desconforto respiratório, tratados com $120 \mathrm{mg} / \mathrm{kg}$ de surfactante porcino. A PEEP inicial foi de $3 \mathrm{~cm} \mathrm{H}_{2} \mathrm{O}$. Para a avaliação da função pulmonar, foi utilizado um pneumotacógrafo com monitor gráfico. Após cada aumento da PEEP ( 4 e $6 \mathrm{~cm}$ de $\mathrm{H}_{2} \mathrm{O}$ ), aguardava-se 20 minutos para se aferir os dados da função pulmonar e gases arteriais.

Resultados: dos 11 pacientes estudados com síndrome do desconforto respiratório, três eram do sexo masculino e oito do sexo feminino $(1: 2,7)$. A idade gestacional foi de 30,78 $\pm 2,05$ semanas, com uma variação de 26 a 34 semanas. O aumento da PEEP de 3 para $6 \mathrm{~cm}$ de $\mathrm{H}_{2} \mathrm{O}$ ocasionou queda significativa do $\mathrm{V}_{\text {Tinsp }}(6,46 \pm 3,43$ para $4,20 \pm$ $2,35, \mathrm{p}=0,0262$ ). Com o aumento da PEEP de 4 para $6 \mathrm{cmH}_{2} \mathrm{O}$, também ocorreu queda do $\mathrm{V}_{\text {Tinsp }}(5,98 \pm 3,33$ para $4,20 \pm 2,35, \mathrm{p}=$ $0,0044)$. Em relação à Cdyn, quando o aumento da PEEP foi de 3 para $6 \mathrm{~cm}$ de $\mathrm{H}_{2} \mathrm{O}$, a diminuição foi estatisticamente significante $(0,58 \pm$ 0,27 para $0,46 \pm 0,25, \mathrm{p}=0,0408$ ), e quando foi de 4 para $6 \mathrm{~cm}$ de $\mathrm{H}_{2} \mathrm{O}$, a diminuição da Cdyn também foi importante $(0,77 \pm 0,27$ para 0,46 $\pm 0,25, \mathrm{p}=0,0164)$. Aumentos da PEEP de 4 para $6 \mathrm{~cm}$ de $\mathrm{H}_{2} \mathrm{O}$ provocaram aumentos nas $\mathrm{PaCO}_{2}(52,81 \pm 15,49$ para $64,90 \pm 12,69$, $\mathrm{p}=0,0141)$. Um aumento mais acentuado foi observado quando a PEEP foi aumentada de 3 para $6 \mathrm{~cm}$ de $\mathrm{H}_{2} \mathrm{O}(41,45 \pm 7,87$ para 64,90 $\pm 12,69, \mathrm{p}=0,0033$ ).

Conclusões: o estudo evidenciou que PEEP de 3 e $4 \mathrm{cmH}_{2} \mathrm{O}$ produzem melhores resultados em termos de Cdyn e menores efeitos colaterais em termos de acidose respiratória e hiperinsuflação pulmonar, com diminuição da ventilação alveolar, evidenciados pelo aumento da $\mathrm{PaCO}_{2}$ e diminuição do $\mathrm{V}_{\text {Tinsp }}$.

$J$ Pediatr (Rio J) 2002; 78 (5): 403-8: PEEP, complacência dinâmica, volume corrente inspiratório, $\mathrm{PaCO}_{2}, \mathrm{SDR}$, recém-nascido pré-termo.

\begin{abstract}
Objective: to verify the alterations of pulmonary function in preterm newborn infants with respiratory distress syndrome (RDS). The parameters analyzed were Dynamic Compliance (Cdyn), Inspiratory Tidal Volume (TVinsp), partial pressure of carbon dioxide $\left(\mathrm{PaCO}_{2}\right)$.

Methods: eleven preterm newborn infants, with gestational age $<$ 35 weeks, and birth weight $<2.500 \mathrm{~g}$, were include in a control case study. All infants presented RDS and were treated with $120 \mathrm{mg} / \mathrm{Kg}$ of porcine surfactant. The initial positive end expiratory pressure (PEEP) was $3 \mathrm{~cm} \mathrm{H}_{2} \mathrm{O}$. A pneumotacograph with a graphical monitor was used to assess the pulmonary function. After each increase in the PEEP (4 and $6 \mathrm{~cm} \mathrm{H}_{2} \mathrm{O}$ ), there was an interval of 20 minutes before measuring the arterial data of pulmonary function and arterial gases.
\end{abstract}

Results: there were three males and eight females (1:2,7) among the infants with RDS. The mean gestational age was 30.78. \pm 2.05 weeks, ranging from 26 to 34 weeks. The increase in the PEEP from 3 to $6 \mathrm{~cm} \mathrm{H}_{2} \mathrm{O}$ caused significant decrease in the TVinsp (6.46 \pm 3.43 to $4.20 \pm 2.35, P=0.0262$ ). With the increase in the PEEP from 4 to 6 $\mathrm{cm} \mathrm{H}_{2} \mathrm{O}$, there was also a decrease in the TVinsp (5.98. \pm 3.33 to 4.20. $\pm 2.35),(P=0.0044)$. Regarding the $\mathrm{Cdyn}$, when there was an increase in the PEEP from 3 to $6 \mathrm{~cm} \mathrm{H}_{2} \mathrm{O}$, the reduction was statistically significant $(0.58 . \pm 0.27$ to $0.46 \pm 0.25, P=0.0408)$ and from 4 to $6 \mathrm{~cm}$ $\mathrm{H}_{2} \mathrm{O}$, the reduction in the Cdyn was also important $(0.77 \pm 0.27$ to 0.46 $\pm 0.25),(P=0.0164)$. Increases in the PEEP from 4 to $6 \mathrm{~cm} \mathrm{H}_{2} \mathrm{O}$ caused increases in the $\mathrm{PaCO}_{2}(52.81 \pm 15.49$ to $64.90 \pm 12.69),(P=0,0141)$. A more significant increase was observed when the PEEP was elevated from 3 to $6 \mathrm{~cm} \mathrm{H}_{2} \mathrm{O}(41.45 \pm 7.87$ to $64.90 \pm 12.69, P=0.0033)$.

Conclusions: the study showed that the PEEP from 3 to $4 \mathrm{~cm} \mathrm{H}_{2} \mathrm{O}$ produces better results in terms of Cdyn and less collateral effects regarding respiratory acidosis and pulmonary hyperventilation with impairment of the alveolar ventilation, evidenced by the increase in the $\mathrm{Pa}_{\mathrm{CO} 2}$ and the decrease in the TVinsp.

JPediatr (Rio J) 2002; 78 (5): 403-8: peep, dynamic compliance, inspiratory tidal volume, $\mathrm{PaCO}_{2}$, RDS, preterm newborn.

1. Professor Auxiliar do Departamento de Pediatria - Universidade Federal de Mato Grosso do Sul (UFMS). Mestre em Medicina - Pediatria.

2. Professor Adjunto Doutor do Departamento de Pediatria - UFMS

3. Professora Adjunta do Departamento de Pediatria - UFMS. Mestre em Medicina - Pediatria.

Artigo submetido em 31.01.02, aceito em 19.06.02. 


\section{Introdução}

A síndrome do desconforto respiratório (SDR), mais do que um processo de doença é um distúrbio do desenvolvimento, geralmente associada com o nascimento prematuro, e apesar dos grandes avanços que ocorreram para se entender a fisiopatologia da SDR e o papel que o surfactante desempenha como causa, permanece o problema clínico principal e uma das mais comuns causas de morbidade em recém-nascidos pré-termo ${ }^{1}$ (RNPT). De todos os problemas respiratórios que afetam o recém-nascido $(\mathrm{RN})$, a doença da membrana hialina constitui um dos mais graves e freqüentes. Cerca de $50 \%$ dos óbitos que ocorrem no período neonatal estão relacionados a distúrbios respiratórios, participando a SDR em cerca de 80 a $90 \%$ dos casos, durante a primeira semana de vida ${ }^{2}$.

Cerca de 30\% dos RNPT com SDR geralmente requerem o uso de pressão de distensão contínua, quer na forma de pressão positiva contínua na via aérea (CPAP), quer através de entubação traqueal com pressão positiva no final da expiração (PEEP). No tratamento ao RNPT com doença da membrana hialina, principalmente nos que são submetidos à ventilação mecânica, uma monitorização mais intensa, sobretudo da mecânica respiratória, deve ser realizada. Análise do fluxo e do volume, através de pneumotacógrafo, é amplamente utilizada. As curvas pressão-volume também são determinadas por esses equipamentos. A complacência dinâmica, o volume corrente inspiratório e expiratório e a perda de ar do sistema podem ser avaliados contínua e simultaneamente ${ }^{3,4}$. Desta forma, consegue-se minimizar os efeitos colaterais da ventilação mecânica, do próprio uso da PEEP, prevenindo-se danos pulmonares (barotraumas e volotrauma), danos cardiocirculatórios e sistêmicos.

O presente estudo teve como objetivo avaliar as alterações da função pulmonar: complacência dinâmica (Cdyn), volume corrente inspiratório $\left(\mathrm{V}_{\text {Tinsp }}\right)$ e alterações da pressão arterial de dióxido de carbono $\left(\mathrm{PaCO}_{2}\right)$ em recémnascidos pré-termo com síndrome do desconforto respiratório, com PEEP de 3, 4 e $6 \mathrm{~cm}$ de $\mathrm{H}_{2} \mathrm{O}$.

\section{Pacientes e métodos}

Foram estudados 11 RNPT com idade gestacional inferior a 35 semanas, avaliados pelo método de Ballard modificado $^{5}$, e com peso de nascimento menor que 2.500 gramas. Todos tinham diagnóstico clínico e radiológico de SDR. Todos pacientes estavam internados na UTI neonatal do Hospital Universitário da Universidade Federal de Mato Grosso do Sul (HU-UFMS), estavam sob ventilação mecânica e foram submetidos ao estudo após apresentar dados clínicos e gases sangüíneos estáveis por, no mínimo, duas horas após a administração do surfactante.

A permissão de um dos pais foi obtida, e o termo de consentimento foi assinado, para a participação do estudo. O protocolo para a pesquisa foi aprovado pela Comissão de Ética Médica da UFMS.
Todos os RNs eram sedados e curarizados quando necessário, para que a respiração espontânea e/ou o esforço respiratório não interferisse nas aferições da função pulmonar. Crianças portadoras de doenças cardíacas, pulmonares, neuromusculares, renais, malformações, com distúrbios hidroeletrolíticos, metabólicos ou cardiocirculatórios não participaram do estudo.

O diagnóstico clínico adotado foi baseado nos critérios adotados por Walter e Taeusch ${ }^{6}$, que incluem dados clínicos e radiológicos.

Logo após o diagnóstico da SDR, o surfactante porcino $\left(\right.$ Curosurf $\left.^{\circledR}\right)$ foi usado na dose de $120 \mathrm{mg} / \mathrm{kg}$ de peso $(1,5 \mathrm{ml}$ é igual a $120 \mathrm{mg}$ ). De acordo com a evolução clínica e dos gases sangüíneos do paciente, uma segunda dose foi realizada.

A artéria umbilical foi cateterizada apropriadamente. Os gases sangüíneos foram medidos a partir de uma amostra de sangue heparinizado (50 unidades) em seringa para insulina. A análise foi feita em um aparelho Radiometer ABL 330, para os diferentes níveis da PEEP $\left(3 \mathrm{cmH}_{2} \mathrm{O}, 4\right.$ $\mathrm{cmH}_{2} \mathrm{O}$ e $6 \mathrm{cmH}_{2} \mathrm{O}$ ), com intervalo de 20 minutos entre cada uma. A oximetria de pulso foi realizada por um oxímetro digital da marca Ohmeda, de modo contínuo, desde a chegada do paciente até o final da coleta dos gases sangüíneos arteriais. O transdutor do oxímetro de pulso foi colocado ao nível de polegar direito.

$\mathrm{O}$ respirador é do tipo ciclado a tempo com pressão limitada - SECHRIST IV 100B (Sechrist, Ana Heim, California, USA). Os parâmetros ventilatórios iniciais (PIP, FR, TI, TE, relação $\mathrm{I} / \mathrm{E} \mathrm{e} \mathrm{FiO}_{2}$ ) foram ajustados com o objetivo de manter os gases arteriais em valores pré-determinados: $\mathrm{pH}$ entre 7,20 e 7,407; $\mathrm{PaCo}_{2}$ entre 30 e $50 \mathrm{mmHg} ; \mathrm{PaO}_{2}$ entre 50 e $80 \mathrm{mmHg}$, e a Saturação da $\mathrm{Hb} \geq 90 \%$. A PEEP inicial foi de $3 \mathrm{~cm} \mathrm{H}_{2} \mathrm{O}$.

Após a estabilização do paciente pelo menos por duas horas após o surfactante, os níveis da PEEP eram alterados para 4 e $6 \mathrm{~cm} \mathrm{H}_{2} \mathrm{O}$. Aguardava-se vinte minutos para nova avaliação dos gases sangüíneos entre um aumento e outro da PEEP.

Com exceção da $\mathrm{FiO}_{2}$, para garantir saturação acima de $90 \%$, os demais parâmetros do ventilador não foram alterados para se eliminar variáveis de confundimento. Cada nível da PEEP era mantido por 20 minutos e, no final deste período, os gases arteriais $\left(\mathrm{PaCO}_{2}\right.$ e $\left.\mathrm{PaO}_{2}\right)$ foram analisados.

Para a avaliação da função pulmonar, foi utilizado um pneumotacógrafo com monitor gráfico, NewPort Navigator GM-250, (NMI Newport Medical Instruments, INC, USA), acoplado a um transdutor de fluxo Varfley-Bicore. Não foi permitido escape de ar através das paredes laterais da cânula, o que era avaliado pelo próprio pneumotacógrafo (existência ou não de escape detectado pelo pneumotacógrafo). Foram, então, medidos os seguintes parâmetros, para cada nível da PEEP, após 20 minutos para a estabili- 
zação do paciente: a complacência dinâmica (Cdyn), em mililitros/centímetros de $\mathrm{H}_{2} \mathrm{O}$, e o volume corrente inspiratório $\left(\mathrm{V}_{\text {Tins }}\right)$, em litros.

A análise estatística dos resultados foi feita através da média, do desvio-padrão e da porcentagem. $\mathrm{O}$ teste de Shapiro-Wik foi utilizado para verificar a normalidade das variáveis. O teste dos sinais dos postos de Wilcoxon ${ }^{8}$ (Wilcoxon apud Daniel, 1995) foi usado para comparar as diferenças entre os grupos, antes e depois dos aumentos da PEEP. O valor $\mathrm{p}$ considerado significativo foi menor que 0,05 . Todos os testes estatísticos foram realizados através do aplicativo Microsoft ${ }^{\circledR}$ Excel 4.0, licenciado para o Centro de Aprimoramento Pediátrico (CAPE), do Departamento de Pediatria da UFMS, sob a identificação número 61.616, e do programa Stataquest 4.0 para Windows 95, número de série: W-902103040, Stata Corporation Tx, USA.

\section{Resultados}

Dos onze pacientes estudados com SDR, três eram do sexo masculino e oito do sexo feminino $(1: 2,7)$. A idade gestacional foi de $30,78 \pm 2,05$ semanas, com uma variação de 26 a 34 semanas. O peso de nascimento variou de 860 a 2.270 gramas, com uma média e desvio-padrão de 1.489,10 $\pm 358,28$ gramas (média e desvio-padrão). O tempo médio em que foi administrada a primeira dose de surfactante exógeno foi de 7,5 $\pm 6,54$ horas, com uma variação de 1,5 a 24 horas.

Para os níveis da PEEP de 3, 4 e $6 \mathrm{cmH}_{2} \mathrm{O}$, os valores correspondentes ao volume corrente inspiratório $\left(\mathrm{V}_{\text {Tins }}\right)$, complacência dinâmica (Cdyn) e pressão parcial arterial de dióxido de carbono $\left(\mathrm{PaCO}_{2}\right)$ estão expressos na Tabela 1.

Com o aumento da PEEP de 3 para $4 \mathrm{~cm} \mathrm{de}_{2} \mathrm{O}$, ocorreu uma redução nos valores do $\mathrm{V}_{\text {Tinsp }}$ de 6,46 $\pm 3,43$ para 5,98 $\pm 3,33$, respectivamente. Porém esta diferença não foi estatisticamente significante $(\mathrm{p}=0,4234)$. Já o aumento da PEEP de 3 para $6 \mathrm{~cm}$ de $\mathrm{H}_{2} \mathrm{O}$ ocasionou uma queda estatisticamente significante do $\mathrm{V}_{\text {Tins }}(6,46 \pm 3,43$ para $4,20 \pm$ $2,35, \mathrm{p}=0,0262)$. Com o aumento da PEEP de 4 para 6 $\mathrm{cmH}_{2} \mathrm{O}$, também ocorreu uma queda estatisticamente significante do $\mathrm{V}_{\text {Tins }}(5,98 \pm 3,33$ para $4,20 \pm 2,35, \mathrm{p}=0,0044)$.

Em relação à complacência dinâmica, os efeitos do aumento da PEEP de 3 para $4 \mathrm{~cm}$ de $\mathrm{H}_{2} \mathrm{O}$, não houve alterações estatisticamente significantes $(\mathrm{p}=0,5045)$. Quando o aumento da PEEP foi de 3 para $6 \mathrm{~cm}$ de $\mathrm{H}_{2} \mathrm{O}$, a diminuição da Cdyn foi estatisticamente significante $(0,58$ $\pm 0,27$ para $0,46 \pm 0,25, \mathrm{p}=0,0408)$, e de 4 para $6 \mathrm{~cm}$ de $\mathrm{H}_{2} \mathrm{O}$, a diminuição da Cdyn também foi importante $(0,77 \pm$ $0,27$ para $0,46 \pm 0,25, \mathrm{p}=0,0164)$.

Tabela 1 - Valores dos parâmetros da função pulmonar e da $\mathrm{PaCO}_{2}$, obtidos com nível da PEEP em 3 , 4 e 6 cmH $\mathrm{C}_{2} \mathrm{O}$, dos recémnascidos com SDR estudados

\begin{tabular}{|c|c|c|c|c|c|c|c|c|c|}
\hline \multirow[t]{2}{*}{ Pacientes } & \multicolumn{3}{|c|}{ Cdyn* } & \multicolumn{3}{|c|}{$\mathbf{V}_{\text {Tinsp }}^{\dagger}$} & \multicolumn{3}{|c|}{$\mathrm{PaCO}_{2}^{\ddagger}$} \\
\hline & PEEP3 & PEEP4 & PEEP6 & PEEP3 & PEEP4 & PEEP6 & PEEP3 & PEEP4 & PEEP6 \\
\hline 1 & 0,31 & 0,28 & 0,18 & 1,6 & 2,2 & 1,9 & 50 & 56 & 56 \\
\hline 2 & 0,87 & 0,78 & 0,55 & 7,6 & 7 & 3,7 & 45 & 45 & 64 \\
\hline 3 & 0,24 & 0,34 & 0,39 & 3,4 & 2,4 & 2,6 & 34 & 79 & 64 \\
\hline 4 & 1,1 & 1,27 & 0,80 & 12,1 & 10,1 & 5,5 & 40 & 43 & 60 \\
\hline 5 & 0,92 & 1,02 & 1,04 & 7,5 & 5,3 & 5,0 & 32 & 70 & 85 \\
\hline 6 & 0,56 & 0,62 & 0,45 & 6,5 & 6,4 & 4,7 & 41 & 43 & 58 \\
\hline 7 & 0,39 & 2,39 & 0,25 & 3,3 & 3,5 & 1,6 & 36 & 37 & 54 \\
\hline 8 & 0,63 & 0,63 & 0,42 & 7,0 & 6,6 & 4,8 & 44 & 30 & 51 \\
\hline 9 & 0,44 & 0,30 & 0,33 & 6,2 & 6,7 & 5,7 & 59 & 56 & 67 \\
\hline 10 & 0,58 & 0,56 & 0,4 & 12,7 & 12,9 & 9,4 & 40 & 50 & 63 \\
\hline 11 & 0,41 & 0,33 & 0,28 & 3,7 & 2,7 & 1,3 & 35 & 72 & 92 \\
\hline Média & $0,58 \pm$ & $0,77 \pm$ & $0,46 \pm$ & $6,46 \pm$ & $5,98 \pm$ & $4,20 \pm$ & $41,45 \pm$ & $52,81 \pm$ & $64,90 \pm$ \\
\hline e DP & 0,77 & 0,62 & 0,25 & 3,43 & 3,33 & 2,35 & 7,87 & 15,49 & 12,69 \\
\hline
\end{tabular}

* complacência dinâmica, † volume corrente inspiratório, ‡ pressão arterial de dióxido de carbono.

PEEP de 3 para $6 \mathrm{~cm}$ de $\mathrm{H}_{2} \mathrm{O}$, queda da $\mathrm{V}_{\text {Tins }}(\mathrm{p}=0,0262)$.

PEEP de 4 para $6 \mathrm{cmH}_{2} \mathrm{O}$, queda $\mathrm{V}_{\text {Tins }}(5,98 \pm 3,33$ para $4,20 \pm 2,35, \mathrm{p}=0,0044)$.

PEEP 3 para $6 \mathrm{~cm}$ de $\mathrm{H}_{2} \mathrm{O}$, diminuição da Cdyn ( $\left.p=0,0408\right)$; 4 para $6 \mathrm{~cm}$ de $\mathrm{H}_{2} \mathrm{O}$, diminuição da $\mathrm{Cdyn},(\mathrm{p}=0,0164)$.

PEEP 4 para $6 \mathrm{~cm}$ de $\mathrm{H}_{2} \mathrm{O}$, aumento $\mathrm{PaCO}_{2}(p=0,0141)$; PEEP de 3 para $6 \mathrm{~cm}$ de $\mathrm{H}_{2} \mathrm{O}(\mathrm{p}=0,0033)$. 
Incrementos das pressões positivas no final da expiração de 3 para $4 \mathrm{~cm}$ de $\mathrm{H}_{2} \mathrm{O}$ não levaram a alterações nas $\mathrm{PaCO}_{2}(41,45 \pm 7,87$ para $52,81 \pm 15,49, \mathrm{p}=0,0749)$. Aumentos de 4 para $6 \mathrm{~cm}$ de $\mathrm{H}_{2} \mathrm{O}$ provocaram aumentos estatisticamente significantes nas $\mathrm{PaCO}_{2}(52,81 \pm 15,49$ para $64,90 \pm 12,69, \mathrm{p}=0,0141)$. Um aumento mais acentuado foi observado quando a PEEP foi aumentada de 3 para $6 \mathrm{~cm} \mathrm{de} \mathrm{H}_{2} \mathrm{O}(41,45 \pm 7,87$ para $64,90 \pm 12,69, \mathrm{p}=0,0033)$.

\section{Discussão}

Na ventilação mecânica do RN pré-termo com SDR, mesmo com uso do surfactante, a PEEP é largamente usada para prevenir o colapso alveolar, manter a capacidade residual funcional (CRF) e melhorar a oxigenação. A PEEP refere-se à manutenção de uma pressão positiva na via aérea durante a expiração, associada a uma ventilação mecânica intermitente com pressão positiva ${ }^{2}$. Isso resulta em uma melhor distribuição da ventilação em regiões com uma baixa relação $\mathrm{V} / \mathrm{Q}$ (ventilação perfusão) dos pulmões e em um melhor equilíbrio $\mathrm{V} / \mathrm{Q}^{9-11}$. Portanto, a PEEP tem uma importância primária na melhora da oxigenação, na diminuição da necessidade de altas concentrações de $\mathrm{O}_{2}$ inspirado, aumento da CRF e melhora da complacência respiratória total nos casos de doenças associadas com o decréscimo do volume residual e da complacência.

Falke et al. ${ }^{12}(1972)$, estudando dez recém-nascidos com insuficiência respiratória aguda sob ventilação mecânica, verificaram os efeitos da PEEP de 0,10 e $15 \mathrm{~cm}$ de $\mathrm{H}_{2} \mathrm{O}$ aplicados a cada 30 minutos. A Cdyn decresceu progressivamente com o aumento da PEEP $(\mathrm{p}<0,01)$, e ocorreu um aumento na $\mathrm{PaO}_{2}$ com os níveis da PEEP de 5 a $15 \mathrm{~cm}$ de $\mathrm{H}_{2} \mathrm{O}(\mathrm{p}<0,01)$. A queda na Cdyn reflete um aumento da rigidez dos alvéolos altamente distendidos. Por outro lado, o aumento na $\mathrm{PaO}_{2}$ pode indicar uma simultânea ocorrência de recrutamento de espaços aéreos associados à hiperinsuflação de alvéolos previamente abertos. Philips III et al. ${ }^{4}$ (1980) avaliaram a Cdyn em 24 pré-termos com SDR, relacionados com PEEP, e observaram decréscimos significativos $(p<0,05)$ para cada aumento da PEEP. No presente estudo, os efeitos do aumento da PEEP de 3 para $4 \mathrm{~cm}$ de $\mathrm{H}_{2} \mathrm{O}$ não causaram alterações significativas da Cdyn $(p=0,5045)$. Com o aumento da PEEP de 3 para $6 \mathrm{~cm}$ $\mathrm{de}_{2} \mathrm{O}$, houve uma diminuição estatisticamente significante da Cdyn ( $\mathrm{p}=0,0408)$, como também com a PEEP de 4 para $6 \mathrm{~cm}$ de $\mathrm{H}_{2} \mathrm{O}(\mathrm{p}=0,0164)$.

A queda da Cdyn poderia ser explicada pelo fato de que a aplicação de uma PEEP maior que 3 ou $4 \mathrm{~cm}$ de $\mathrm{H}_{2} \mathrm{O}$ poderia levar a uma distensão alveolar e a um aumento da CRF. Os alvéolos mais distendidos têm uma maior pressão de recuo elástico e, por compartilharem de uma via aérea comum, algum ar tende a seguir o gradiente de pressão e mover-se para outros alvéolos. Presumivelmente, esse limite representa o ponto no qual o recrutamento máximo do espaço aéreo foi atingido, e aumentos adicionais na PEEP irão meramente sobredistender os espaços aéreos já abertos. A partir daí, ocorreria diminuição da Cdyn ${ }^{12-13}$.
Dimitrou et al. ${ }^{2}$ (1999), estudando oito prematuros, encontraram valores médios da Cdyn para três valores da PEEP. Field et al. ${ }^{15}$ (1985) avaliaram 15 pacientes com SDR e observaram as variações da Cdyn relacionadas com as variações da PEEP. Quando comparados os resultados deste estudo com os valores da literatura, apenas os dados da PEEP de $6 \mathrm{cmH}_{2} \mathrm{O}$ são semelhantes aos encontrados por Dimitrou et al. ${ }^{2}$ (1999). Comparado aos valores encontrados por Graff ${ }^{16}$ (1986), os valores são muito semelhantes, principalmente nos níveis de 3 e $4 \mathrm{cmH}_{2} \mathrm{O}$ de PEEP e aos níveis obtidos por Field et al. ${ }^{15}$ (1985).

Pelos dados obtidos no presente estudo, a melhor média para a Cdyn foi com uma PEEP de $4 \mathrm{cmH}_{2} \mathrm{O}(0,77 \pm 0,62)$. Segundo estudo de Cheifetz et al. ${ }^{14}$ (1998), uma queda maior ou igual a $20 \%$ na Cdyn indica a presença de hiperinsuflação pulmonar. As maiores diminuições da Cdyn ocorreram entre o aumento da PEEP de 3 para $6 \mathrm{cmH}_{2} \mathrm{O}$ $(21,42 \%)$ e aumento da PEEP de 4 para $6 \mathrm{cmH}_{2} \mathrm{O}(40,25 \%)$, explicando as outras alterações observadas pela provável hiperinsuflação pulmonar.

Evidenciou-se, pois, que uma PEEP adequada previne o colapso alveolar, mantendo um volume no final da expiração, e melhora a relação $\mathrm{V} / \mathrm{Q}$, aumentando, assim, a $\mathrm{PaO}_{2}$. Em face das variações na PEEP alterarem o gradiente de pressão entre inspiração e expiração, a eliminação do dióxido de carbono $\left(\mathrm{CO}_{2}\right)$ pode ser afetada. Desse modo, uma elevação na PEEP pode diminuir o volume corrente $\mathrm{e}$ a eliminação de $\mathrm{CO}_{2}$, levando a um aumento na pressão parcial arterial do dióxido de carbono $\left(\mathrm{PaCO}_{2}\right)^{17}$. Portanto, hipoventilação alveolar deveria ocorrer após aumento da PEEP, seguida por um aumento correspondente na $\mathrm{PaCO}_{2}$. Assim, um decréscimo na PEEP deveria ser considerado quando ocorresse retenção de $\mathrm{CO}_{2}$, principalmente se a hipoxemia não for problema. No presente estudo, o incremento da PEEP de 3 para $4 \mathrm{~cm}$ de $\mathrm{H}_{2} \mathrm{O}$ não levou a alterações significativas na $\mathrm{PaCO}_{2}(41,45 \pm 7,87$ para 52,81 $\pm 15,49, \mathrm{p}=0,0749$ ). Aumentos de 4 para $6 \mathrm{~cm}$ de $\mathrm{H}_{2} \mathrm{O}$ provocaram aumento estatisticamente significante na $\mathrm{PaCO}_{2}$ $(52,81 \pm 15,49$ para $64,90 \pm 12,69, \mathrm{p}=0,0141) . \mathrm{Um}$ aumento mais acentuado foi observado quando a PEEP foi aumentada de 3 para $6 \mathrm{~cm}$ de $\mathrm{H}_{2} \mathrm{O}(41,45 \pm 7,87$ para 64,90 $\pm 12,69, \mathrm{p}=0,0033)$.

Uma outra conseqüência da diminuição do volume corrente e, conseqüentemente, da ventilação alveolar, seria a retenção de $\mathrm{CO}_{2}$ e provável acidose respiratória. Tem sido demonstrado que, após aumentos da PEEP de zero a $6 \mathrm{~cm}$ de $\mathrm{H}_{2} \mathrm{O}$, haveria quedas no $\mathrm{pH}$ em média $7,33(7,3-7,43)$ com PEEP de $3 \mathrm{~cm}$ de $\mathrm{H}_{2} \mathrm{O}$, e média de $7,29(7,16$ a 7,38$)$ para PEEP de $6 \mathrm{cmH}_{2} \mathrm{O}^{2}$.

No mesmo sentido, Bartolomew et al. ${ }^{17}$ (1999) observaram que a queda no volume corrente $\left(\mathrm{V}_{\mathrm{T}}\right)$ diminui o volume minuto, e que também tem implicações nas trocas do $\mathrm{CO}_{2}$. Uma redução na PEEP de $2 \mathrm{cmH}_{2} \mathrm{O}$ aumentou em $30 \%$ o volume corrente. Esse efeito é determinado pela ventilação/ minuto de acordo com a equação do ar alveolar: $\mathrm{PaCO}_{2}=$ 
$\mathrm{V}_{\mathrm{C}}$ / Volume minuto $+\mathrm{K}$, onde $\mathrm{K}$ é uma constante. $\mathrm{A} \mathrm{VCO}_{2}$ é a freqüência de produção do $\mathrm{CO}_{2}$. Isso se aplica especialmente aos recém-nascidos prematuros extremos, nos quais a ventilação alveolar pode já estar comprometida por um espaço anatômico desproporcionadamente grande. A razão do volume do espaço morto e do $\mathrm{V}_{\mathrm{T}}$ é uma importante determinante da ventilação alveolar, e pode ser significativamente melhorada pelo aumento do $\mathrm{V}_{\mathrm{T}}$ ou diminuída pela redução do $\mathrm{V}_{\mathrm{T}}$.

Philips III et al. ${ }^{4}$ (1980) também concluíram que, se o volume corrente cair devido ao aumento da PEEP, o volume minuto também cai, e, portanto, mais distensão alveolar e aumento da pressão intratorácica levam à retenção de $\mathrm{CO}_{2}$, ruptura alveolar e/ou diminuição do débito cardíaco.

Neste trabalho, com o aumento da PEEP de 3 para 6, e de 4 para $6 \mathrm{~cm} \mathrm{de} \mathrm{H}_{2} \mathrm{O}$, diminuiu o $\mathrm{V}_{\text {Tinsp }}$ significativamente $(\mathrm{p}=0,0262$ e $\mathrm{p}=0,0044$, respectivamente).

$\mathrm{O}$ estudo, portanto, evidenciou que PEEP de 3 e 4 $\mathrm{cmH}_{2} \mathrm{O}$ produzem melhores resultados em termos de Cdyn, e menores efeitos colaterais em termos de acidose respiratória e hiperinsuflação pulmonar, com diminuição da ventilação alveolar, evidenciados pelo aumento da $\mathrm{PaCO}_{2} \mathrm{e}$ pela diminuição do $\mathrm{V}_{\text {Tinsp. }}$.

\section{Referências bibliográficas}

1. Hack M, Wright LL, Shankaran S, Tyson JE, Horbar JD, Bauer $\mathrm{CR}$, et al. Very low birthweight outcomes of NICHD neonatal network November 1988 - October 1990. Am J Obstet Gynecol 1995;172:457-64.

2. Dimitrou G, Greenough A, Laubsher B. Appropriate positive end expiratory level in surfactant-treated preterm infants. Eur $\mathrm{J}$ Pediatr 1999;158:888-91.

3. Thome U, Töpfer A, Schaller P, Pohlandt F. The effect of positive end expiratory pressure, peak inspiratory pressure, and inspiratory time on functional residual capacity in mechanically ventilated preterm infants. Eur J Pediatr 1998;157:831-7.

4. Philips JB III, Beale EF, Howard JE, Jaeger MJ, Eitzman DV. Effect of positive end-expiratory pressure on dynamic respiratory compliance in neonates. Biol Neonate 1980;38:270-5.

5. Ballard JL, Khoury JC, Wedig K, Wang L, Eilers-Walsman BL, Cip R. New Ballard score, expanded to include extremely premature infants. J Pediatr 1991;119:417-23.

6. Walther FJ, Taeusch W. Pathophysiology of neonatal surfactant insufficiency: clinical aspects. In: Robertson B, vanGolde LMG, Batengurg JJ, editores. Pulmonary surfactant: from molecular biology to clinical practice. $3^{\mathrm{a}}$ ed. Amsterdam: Elsevier; 1992.p.485-523.

7. Piva JP, Filho JPD. Métodos de ventilação mecânica no paciente pediátrico. In: Relatório do Segundo Consenso Brasileiro de Ventilação Mecânica, 1999.p.11-12. [site na internet]. Disponível em: http://www.amib.com.br.
8. Daniel WW. Biostatistics: a foundation for analysis in the health sciences. $6^{\mathrm{a}}$ ed. New York: John Wiley \& Sons; 1995.

9. Pontoppidan H, Geffin B, Lowenstein E. Acute respiratory failure in the adult (Third of three parts). N Engl J Med 1972; 287:799-806

10. Gong H. Positive-pressure ventilation in the adult respiratory distress syndrome. Clin Chest Med 1982;3:69-88.

11. Dantzker DR, Brook CJ, Dehart P, Lynch JP, Weg JG. Ventilationperfusion distributions in the adult respiratory distress syndrome. Am Rev Respir Dis 1979;120:1039-52.

12. Falke KJ, Pontoppidan H, Kumar A, Leith DE, Geffin B, Laver MB. Ventilation with end-expiratory pressure in acute lung disease. J Clin Invest 1972;51:2315-23.

13. Shaffer TH, Koen PA, Moskowitz GD, Fergunson JD, DelivoriaPapadopoulos M. Positive end expiratory pressure: Effects on lung mechanics of premature lambs. Biol Neonate 1978;34:1-10.

14. Cheifetz IM, Craig DM, Quick G, Mcgovern JJ, Canno L, Ungerleider $\mathrm{M}$, et al. Increasing tidal volumes and pulmonary overdistention adversely affect pulmonary vascular mechanics and cardiac output in a pediatric swine model. Crit Care Med 1998;26:710-6.

15. Field D, Milner AD, Hopkin IE. Effects of positive end expiratory pressure during ventilation of the preterm infant. Arch Dis Child 1985;60:843-7. 
16. Graff MA, Novo RP, Diaz M, Smith C, Hiatt IM, Hegyi T. Compliance measurement in respiratory distress syndrome: the prediction of outcome. Pediatr Pulmonol 1986;2:332-6.

17. Bartholomew KM, Brownlee KG, Snowden S, Dear PRF. To PEEP or not to PEEP? Arch Dis Child 1994;70:209-12.
Endereço para correspondência:

Dr. Luiz C.T. Consolo

Rua Alexandre, 378, Giocondo Orsi

CEP 70902-080 - Campo Grande, MS

Fone: (67) 351.8820-E-mail: tesini@nin.ufms.br 